\title{
High-Intensity Chronic Stroke Motor Imagery Neurofeedback Training at Home: Three Case Reports
}

Clinical EEG and Neuroscience $1-10$

(C) EEG and Clinical Neuroscience Society (ECNS) 2017

Reprints and permissions: sagepub.com/journalsPermissions.nav DOI: 10.1 I77/I5500594I77/7398 journals.sagepub.com/home/eeg (S)SAE

\author{
Catharina Zich',2, Stefan Debener, 1,3, Clara Schweinitz', \\ Annette Sterr ${ }^{5}$, Joost Meekes', and Cornelia Kranczioch ${ }^{1,4}$
}

\begin{abstract}
Motor imagery (Ml) with neurofeedback has been suggested as promising for motor recovery after stroke. Evidence suggests that regular training facilitates compensatory plasticity, but frequent training is difficult to integrate into everyday life. Using a wireless electroencephalogram (EEG) system, we implemented a frequent and efficient neurofeedback training at the patients' home. Aiming to overcome maladaptive changes in cortical lateralization patterns we presented a visual feedback, representing the degree of contralateral sensorimotor cortical activity and the degree of sensorimotor cortex lateralization. Three stroke patients practiced every other day, over a period of 4 weeks. Training-related changes were evaluated on behavioral, functional, and structural levels. All 3 patients indicated that they enjoyed the training and were highly motivated throughout the entire training regime. EEG activity induced by $\mathrm{Ml}$ of the affected hand became more lateralized over the course of training in all three patients. The patient with a significant functional change also showed increased white matter integrity as revealed by diffusion tensor imaging, and a substantial clinical improvement of upper limb motor functions. Our study provides evidence that regular, home-based practice of MI neurofeedback has the potential to facilitate cortical reorganization and may also increase associated improvements of upper limb motor function in chronic stroke patients.
\end{abstract}

\section{Keywords}

motor imagery, neurofeedback, stroke, neurorehabilitation, mobile EEG, home-based training

Received January 21, 2017; revised May 23, 2017; accepted May 29, 2017.

\section{Introduction}

Many of the roughly 33 million stroke survivors worldwide per year ${ }^{1}$ do not recover completely, so that stroke is one of the leading causes of disability. ${ }^{2}$ Upper-limb hemiparesis is the most prevalent consequence in stroke survivors ${ }^{3}$ with enduring and disabling effects, causing negative impact on quality of life and independence. One approach aiming to facilitate motor recovery after stroke is mental practice with motor imagery (MI). The theoretical foundation of this approach is the neural simulation of action theory, ${ }^{4}$ which states that executing and imagining the same movement involves similar, overlapping networks. Hence, mental practice with MI may help prevent nonuse driven cortical reorganization, and facilitate compensatory reorganization such that in the long run, motor functions improve. Indeed, a number of studies using mental practice with MI as an add-on to physical therapy suggest that MI supports recovery of upper-limb functioning, ${ }^{5-9}$ resulting in longlasting behavioral improvements. ${ }^{9}$

A more recent development is the combination of MI training with online neurofeedback (NF). This development is closely linked to the notion that feedback in combination with high-intensity task-specific practice is essential for the facilitation of adaptive cortical reorganization. ${ }^{6}$ Accordingly, NF should help to induce adaptive neural plasticity and thereby contribute to restoring lost motor function..$^{10}$ Several studies indicate that MI training in combination with NF can indeed induce positive changes at behavioral, functional, and structural levels. ${ }^{11-15}$ At least 1 study provides clear evidence that

\footnotetext{
'Neuropsychology Lab, Department of Psychology, European Medical School, University of Oldenburg, Oldenburg, Germany

${ }^{2}$ Department of Experimental Psychology, University of Oxford, Oxford, UK ${ }^{3}$ Cluster of Excellence Hearing4all, University of Oldenburg, Oldenburg, Germany

${ }^{4}$ Research Center Neurosensory Systems, University of Oldenburg, Oldenburg, Germany

${ }^{5}$ Brain and Behaviour Research Group, School of Psychology, University of Surrey, Guildford, UK
}

\section{Corresponding Author:}

Cornelia Kranczioch, Neuropsychology Lab, Department of Psychology, University of Oldenburg, Ammerländer Heerstraße I I4-I I8, D-26 I I I

Oldenburg, Germany.

Email: cornelia.kranczioch@uni-oldenburg.de

Full-color figures are available online at http://journals.sagepub.com/home/eeg 
functional changes are more pronounced when $\mathrm{MI}$ is combined with NF as compared with MI without NF. ${ }^{16}$ Moreover, concurrent acquisition of electroencephalogram (EEG) NF and functional magnetic resonance imaging (fMRI) in healthy individuals validated systematic NF effects on cortical activation patterns. ${ }^{17}$

The effectiveness of neurorehabilitation training regimes such as MI NF is difficult to evaluate, in particular because most approaches are technically demanding, and all of them require frequent, regular practice. The latter issue is of particular importance for stroke victims, because frequent laboratory or hospital visits place a large burden on motor impaired individuals. Without experiencing immediate training effects this effort may negatively impact on a patient's training motivation. Recently developed small and wireless EEG systems may help overcome this problem. They are portable and low in cost, ${ }^{18,19}$ perform on par with nonportable EEG systems, ${ }^{20}$ and soon, NF protocols will be available on smartphones. ${ }^{21}$

We investigated whether home-based, frequent MI EEGbased NF training is feasible. Specifically, we examined whether chronic stroke patients can stay motivated over a 4-week training period. In addition, we wanted to know whether they experience NF as helpful for MI training. We designed the MI NF protocol to facilitate stronger cortical activity in the ipsilesional than in the contralesional hemisphere in response to MI of the paretic hand. Frequent, high-intensity MI NF should result in systematic behavioral, functional, and structural improvements. To assess training effects, the home-based training was framed by detailed laboratory-based assessments of motor functions, high-density EEG, structural as well as fMRI, and by diffusion tensor imaging of the corticospinal tract (CST).

\section{Methods}

\section{Participants}

Three individuals with restricted mobility of one upper limb were recruited through a newspaper advertisement (please refer to the Results section for demographic and clinical characteristics). Inclusion criteria were a first-ever, unilateral vascular event, which was at least 6 months ago, and caused permanent motor impairments in the contralesional upper extremity; MRI compatibility; absence of major cognitive deficits as screened with the Mini-Mental Status Examination (MMSE $<25)$; and no depressive symptoms as screened with the Geriatric Depression Scale (GDS <5). Participants gave written informed consent. The local ethics committee of the University of Oldenburg approved the study.

\section{Motor Imagery Neurofeedback Training}

The training sessions were carried out at the patients' home, in the presence of an instructor (CS). They comprised an MI EEG-based NF session every other day, including weekends, for a period of 4 weeks. Cases P20 and P21 completed 14 and case P22 completed 13 training sessions. Fluctuations of time of day when the training was conducted was kept as minimal as possible ( $S D<2.5$ hours). Each session lasted 60 minutes, of which the pre- and postpreparation of the EEG and questionnaires took approximately 25 minutes. The actual MI training lasted approximately 30 minutes, comprising 3 blocks of roughly 8 minutes each with 4-minute breaks in between. Each of the 3 blocks was composed of 20 right- and 20 left-hand trials, which were pseudo-randomly presented using OpenVibe Designer 0.17.1. ${ }^{22}$ Participants were instructed to imagine the kinesthetic sensation of a power grip from the first-person perspective. The MI task interval lasted for 5 seconds, was cued by a fixation-cross 3 seconds before, and was followed by an intertrial interval of 4.5 to 6 seconds in steps of 0.5 seconds (see Figure 1). During the intertrial interval, participants had the opportunity to initiate a 10 -second break via a foot pedal (Supplemental Methods).

EEG was recorded from 24 scalp sites using a small, wireless, head-mounted amplifier (mBrainTrain; Supplemental Methods). In the first block, no NF was given and acquired EEG data were used to derive the necessary parameters for real-time NF delivery in the second block (Supplemental Methods). Similarly, data acquired in the second block served as calibration for the NF in the third block. In blocks 2 and 3 during the 5-second MI interval real-time NF was provided. The NF protocol developed was based on the observation that chronic stroke is characterized by abnormally strong contralesional sensorimotor activation patterns..$^{23,24}$ The aim of the training was therefore to restore original cortical lateralization patterns toward a stronger contralateral than ipsilateral activity when MI is conducted with the affected limb. In order to achieve this, the NF signal consisted of a 2-dimensional visual display (see Figure 1). A ball moving along the vertical and horizontal axis indicated the degree of contralateral eventrelated desynchronization (ERD) and the degree of ERD laterality, the difference between contra- and ipsilateral ERD, respectively (see Figure 1). The exact position of the ball was determined by the classification of spatially weighted logarithmic band-power features in the range of 8 to $30 \mathrm{~Hz}$. Additionally, EEG data were analyzed offline (Supplemental Methods).

\section{Training Evaluation}

Before and after the 4-week MI EEG-based NF training, upperlimb motor performance was assessed using a modified version of the Fugl-Meyer motor assessment (mFMA). ${ }^{25}$ and spasticity in the wrist and elbow joints in extension and flexion evaluated using a modified version of the Ashworth scale (MAS). ${ }^{26}$ Each test was applied to both the affected and the unaffected hand, where the latter served as a control condition. A certified physiotherapist (CS) carried out all motor assessments. To investigate possible experience-driven functional and structural changes of task-specific neural networks a high-density EEG session and a MRI session were scheduled before and after the training. The high-density EEG session comprised one block of attempted movement (AM), one block of MI and one block of 

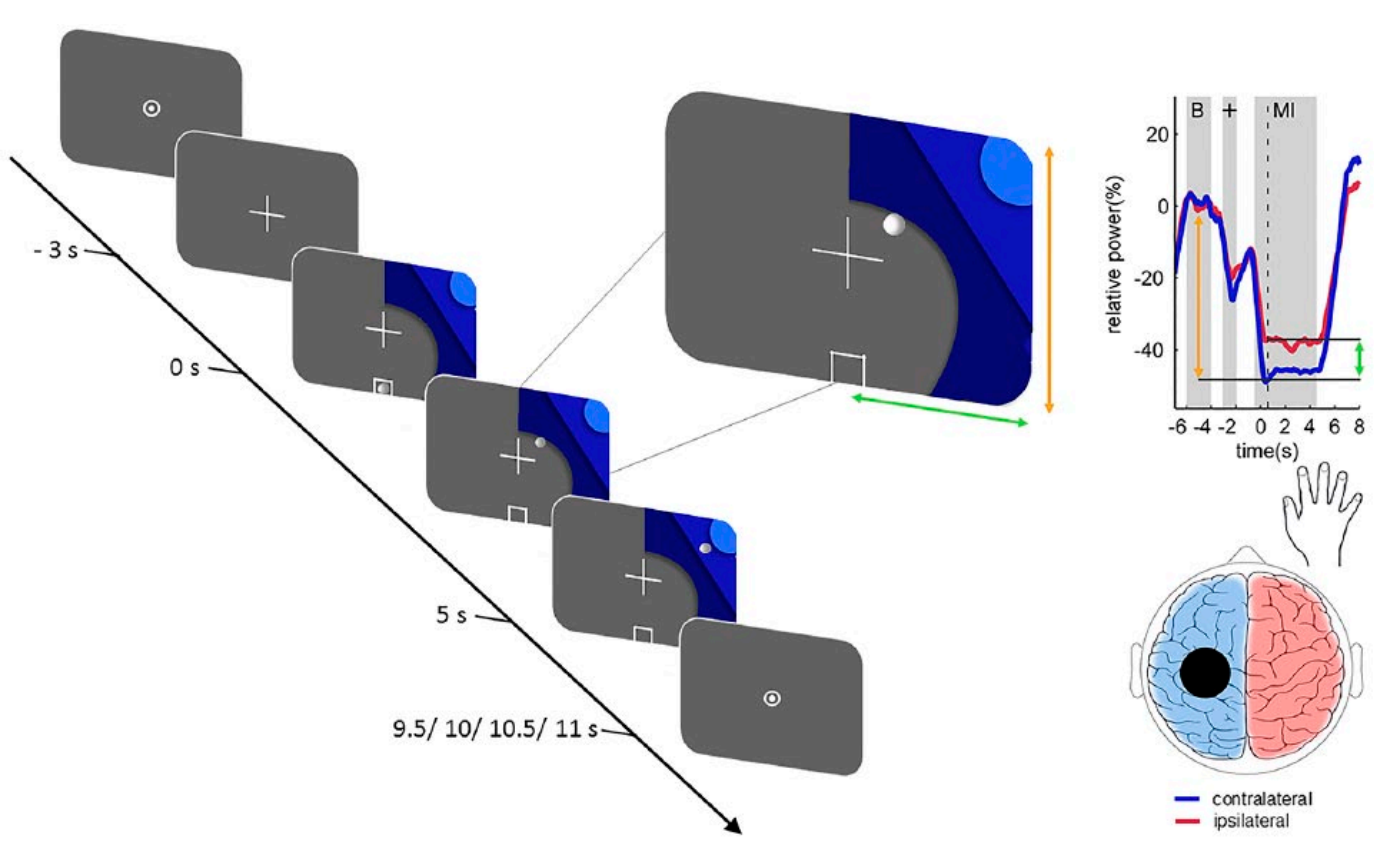

Figure I. Trial structure and relationship between the 2-dimensional neurofeedback (NF) display and motor imagery (MI)-induced brain activity. The trial structure is illustrated exemplary for a right-hand trial. Each trial was initiated with a fixation-cross and after a delay of 3 seconds a graphic comprising 3 different shades of blue was added. Onset of the graphic indicated the beginning of the task period (duration 5 seconds). The location of the graphic indicated which hand to use. During the NF blocks a white circle resembling a ball moved along the horizontal (green arrow) and vertical (orange arrow) axes according to the classifier output magnitudes. Trials were followed by a fixation dot, resulting in an intertrial interval of 4.5 to 6 seconds. The relationship between the position of the ball and the Ml-induced brain activity at the time point of the dashed vertical line is illustrated on an exemplary event-related desynchronization (ERD) time course. The horizontal ball position is determined by the classification of Ml contralateral (blue) versus ipsilateral (red), as illustrated by the green arrow. The vertical ball position is determined by the classification of contralateral baseline ("B") versus contralateral MI, as illustrated by the orange arrow.

MI EEG-based NF, using the same movement and NF procedure as in the home-based sessions. The MRI session included diffusion weighted imaging as well as structural and functional neuroimaging. EEG and MRI data acquisition parameters are provided as Supplemental Methods. The fMRI session comprised one AM and one MI block. For the fMRI session, the inter-trial interval was extended to 5 to 9 seconds, with steps of 1 second, in order to account for the hemodynamic delay, and stimulus presentation was controlled with Presentation software (Neurobehavioral Systems, Albany, NY, USA). To evaluate the MI training, AM-induced fMRI and EEG activity were analyzed and diffusion tensor imaging analysis conducted. Moreover, we performed lesion mapping. Detailed processing steps are provided in the Supplemental Methods. Every session participants were asked to rate the perceived intensity of MI and the support of NF on a 5-point Likert-type scale as well as their motivation on a visual analogue scale. Additionally, comments made spontaneously by the patients were recorded.

\section{Primary and Secondary Outcome Measures}

The primary outcome measure, motor function, was assessed with the mFMA and the MAS. In addition, various secondary outcome measures were investigated. Functional changes were evaluated with MI-induced ERD, AM-induced ERD and AM-related sensorimotor BOLD (blood oxygen level dependent) activity. Structural changes were quantified by means of fractional anisotropy (FA) of the CST. Subjective ratings on motivation and task characteristics, average low-density classification accuracies and patient's comments were jointly considered to judge the feasibility of the MI NF training protocol. All primary and secondary outcome measures were assessed and evaluated separately for each patient.

\section{Results}

\section{Case I}

P20 was a 71-year-old right-handed retired woman with a right-sided ischemic lesion of the Pons (see Figure 2A). Time since vascular event was 7 months. Lesion size was $0.37 \mathrm{~cm}^{3}$ and lesion-CST overlap was $0.30 \mathrm{~cm}^{3}$, which corresponds to $2.12 \%$ of the CST size. Her initial mFMA score was 52 showing only minor deficits in fine motor skills and minor resistance in wrist and elbow extension resulting in an MAS score of 2 in 

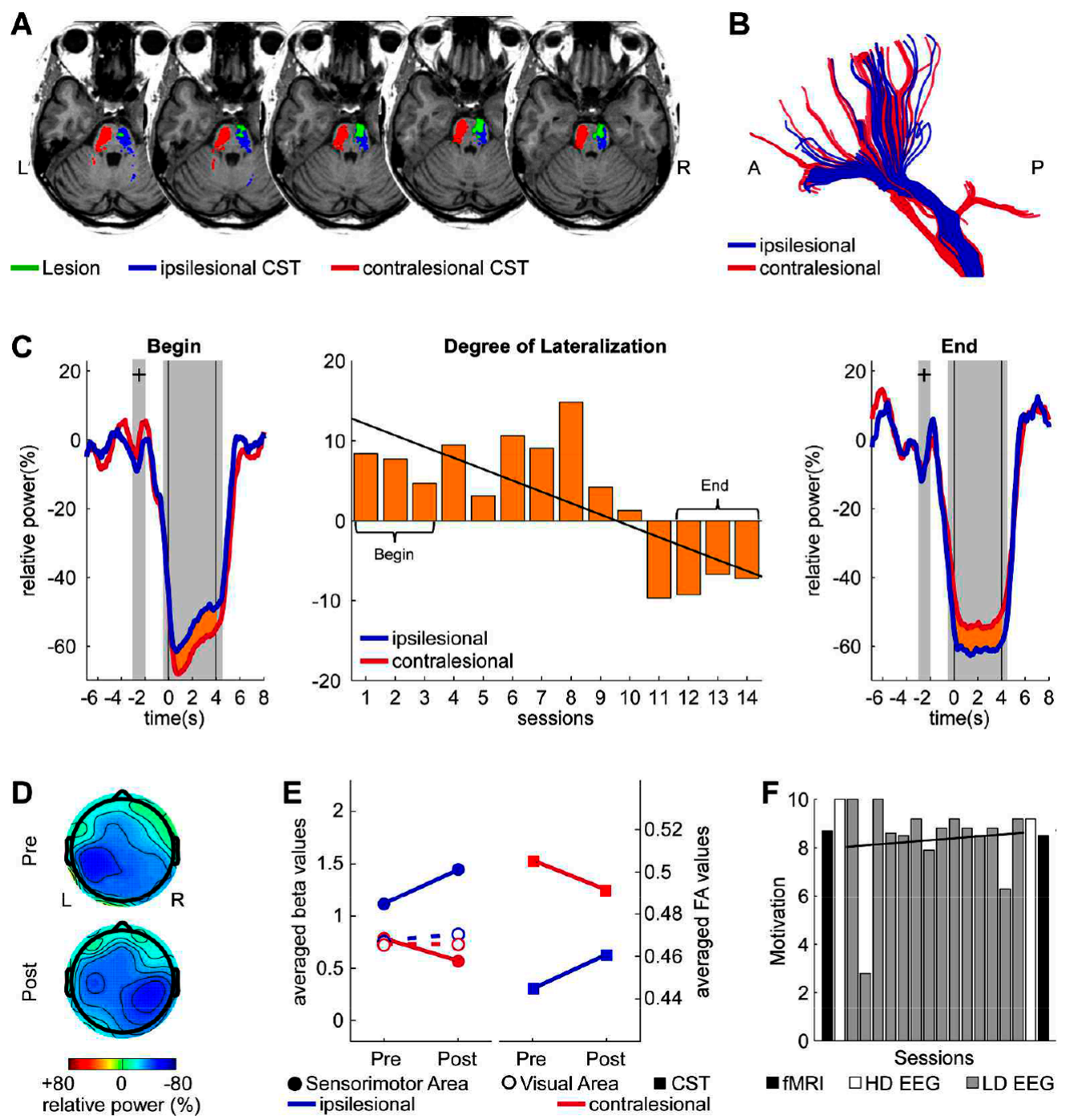

Figure 2. Lesion characteristics and secondary outcome measures P20. (A) Individual lesion map (green) overlaid onto anatomical (Johns Hopkins University [JHU] White-Matter Tractography Atlas) ipsilesional corticospinal tract (CST) (blue) and contralesional CST (red). The axial slices depicted correspond to $z=21,22,23,24$, and 25 in MNI (Montreal Neurological Institute) space. (B) Diffusion tensor imagingbased reconstruction of individual ipsilesional (blue) and contralesional (red) CST fibers illustrated in the sagittal view. (C) Event-related desynchronization (ERD) related to motor imagery (MI) of the affected hand over the training period. Time course of relative power in $\%$ induced by $\mathrm{Ml}$ averaged for the first 3 (left) and last 3 (right) trainings sessions. Means for the ipsilesional hemisphere are visualized in blue and for the contralesional hemisphere in red. The first gray-shaded area indicates the time window of fixation-cross onset, and the second gray-shaded area indicates the time window of MI. The 2 vertical lines indicate the interval $(0.5-4.5$ seconds) used to extract the lateralization parameter (contralateral-ipsilateral ERD) illustrated in the middle. Data from the 2 neurofeedback (NF) blocks were averaged. (D) Topography of ERD induced by attempted movement (AM) of the affected hand before (top) and after (bottom) the MI training assessed with high-density EEG. (E) Functional magnetic resonance imaging (fMRI) activity (left) and averaged fractional anisotropy (FA) values (right) measured before and after the MI training. fMRI blood oxygen level-dependent (BOLD) activity during AM extracted from ipsilesional (blue) and contralesional (red) anatomical region of interests. Brodmann areas I, 2, 3, and 4 were regions of interest (colored circles, solid lines) and Brodmann areas 17 and 18 were control regions (open circles, dashed line). FA values were extracted from individuals contralesional (blue) and ipsilesional (red) CST fibers. (F) Motivation scores obtained on fMRI, high-density (HD) EEG and lowdensity (LD) EEG sessions. Regression lines are fitted for the LD EEG sessions.

her left, nondominant hand. Besides the minor motor deficits of the upper limb, P20 did not show additional motor impairments. Self-report revealed an unsteady gait, which was not obvious by observation. After the intervention, her mFMA score was 59 and her MAS score 0. P20 was medication free during the study. 
P20 had a strong ERD during MI of the affected hand, but at the beginning of the training this ERD was stronger on the contralesional side than ipsilesionally. This reversed activity pattern of stronger contralesional than ipsilesional activity is typical for chronic stroke patients and has been described as detrimental. ${ }^{23}$ Over the training period, the lateralization of the ERD for MI of the affected hand (ipsilesional-contralesional) changed significantly (bootstrapping $1000 ; P<.01$ ) toward a stronger ipsilesional than contralesional activity (see Figure 2C). Pre- and posttraining high-density EEG showed a similar reversal of laterality of the ERD during AM (Figure 2D). Moreover, AM induced stronger lateralization of sensorimotor fMRI BOLD activity after the MI training as compared with before the MI training (Figure 2E). The increased lateralization was attributable to both an increase in ipsilesional and a decreased contralesional activity. These functional changes were accompanied by structural changes: The difference in white matter integrity between the ipsilesional and the contralesional CST decreased, due to an increased FA in the ipsilesional CST and decreased FA in the contralesional CST (Figure 2E). P20 was highly motivated throughout the study, motivation scores showing a positive trend despite slight fluctuations (bootstrapping $1000 ; P>.1$ ). Overall, she rated the MI as moderately intense and the MI NF as providing little support. Spontaneously, she said, that during the MI "... something in my hand was working" and ". . . my hand got warm, because my imagination was so strong." For both NF dimensions, high single trial classification accuracies were obtained across all training sessions (contralateral ERD, $M=88.79 \%, S D=3.48 \%$; ERD laterality, $M=80.39 \%, S D=5.55 \%$ ).

In summary, motor assessment revealed a clinical relevant increase in mFMA scores and a decrease in MAS scores. Moreover, all functional imaging measures revealed increased lateralization during MI and AM of the affected hand. Functional changes were paralleled by structural changes toward a more balanced white matter integrity between left and right CST.

\section{Case 2}

P21 was a 51-year-old right-handed man, who was unemployed. He suffered from a right-sided basal ganglia hemorrhage 30 months before participation in this study. The size of the lesion was $6.29 \mathrm{~cm}^{3}$ and the overlap between lesion and CST was 1.92 $\mathrm{cm}^{3}$ or $14.1 \%$ of the size of the CST (Figure 3A). His initial mFMA score was 10 resulting from residual shoulder and elbow function and he showed severe spasticity in his paralyzed wrist and elbow joints leading to an MAS score of 8 . The stroke resulted in a motor hemiparesis, also causing motor impairments of the lower left limb, although the patient was able to walk unaided. After the intervention, his mFMA score was 11 and the MAS score 7. P21 was medication free during the study.

Overall, the ERD induced by MI of the affected hand, when compared with the ERD of P20 and P22, was of medium strength and in most training sessions ipsilesional ERD was stronger than contralesional ERD. Over the course of MI training, the ERD lateralization increased, although this increase was not significant (bootstrapping 1000; $P>.1$, see Figure 3C). Moreover, P21 showed strong intraindividual variability in ERD lateralization across MI sessions. A pre-post comparison of high-density EEG activity of AM indicated stronger ERD after the training (Figure 3D), while retaining a comparable level of ERD lateralization. On the other hand, sensorimotor fMRI activity induced by AM was slightly increased after the training in the ipsilesional hemisphere, but remained unchanged in the contralesional hemisphere, resulting in a small increase in lateralization (Figure 3E). White matter integrity of the contralesional CST was also unchanged between the pre- and postassessment, FA of the ipsilesional CST was found to be minimally decreased after the MI training (Figure 3E). P21 was highly motivated throughout the study with motivation scores showing a positive trend (bootstrapping $1000 ; P>.1$ ). Overall, he rated the MI as intense and said spontaneously "... a warm flow in the hand." and "... a tingling in my hand, as if someone strokes the skin with a brush." The NF was rated as very supportive and spontaneously described as "Great to see: I moved something!" and "This spurs me on!" For both NF dimensions, moderate single trial classification accuracies were obtained across all training sessions (contralateral ERD, $M=63.51 \%$, $S D=5.53 \%$; ERD laterality, $M=69.03 \%, S D=4.33 \%$ ).

In sum, behavioral outcomes indicate a minor increase in mFMA scores as well as a small decrease in MAS scores. MI induced an ERD of medium strength, which fluctuated largely over the course of training. The small changes observed on functional and structural level are not necessarily facing the same direction.

\section{Case 3}

Participant P22 was a 56-year-old man, retired since the vascular event. He was diagnosed with an ischemic, left-sided infarction of the medial and anterior cerebral arteries resulting in right-sided hemiparesis (Figure 4A), that is, on his premorbidly dominant side. The lesion size was $125.63 \mathrm{~cm}^{3}$ and the lesionCST overlap was $1.31 \mathrm{~cm}^{3}$ or $9.64 \%$ of the size of the CST. The stroke occurred 55 months prior to onset of the study. His initial mFMA score was 3 resulting from residual shoulder function and his MAS score was 8, showing severe motor deficits and high spasticity in his wrist and elbow joints. He was able to walk independently despite the lower limb paresis. Furthermore, he showed an aphasia, which did not affect his understanding or performance of the tasks. Following the intervention, his mFMA and MAS score were unchanged. P22 was on medication during the study (Supplemental Material).

During MI of the affected hand, P22 showed a strong ERD, which was in all training sessions stronger over the ipsilesional hemisphere than over the contralesional hemisphere. During the training, a trend for significantly increased MI-induced ERD lateralization (bootstrapping 1000; $P<.06$ ) could be observed (Figure 4C). In contrast to MI, AM of the affected hand was related to a very small, hardly visible, ERD (Figure 4D). No changes in AM-related ERD strength or lateralization between the 2 different times of measurement were observed, 
A

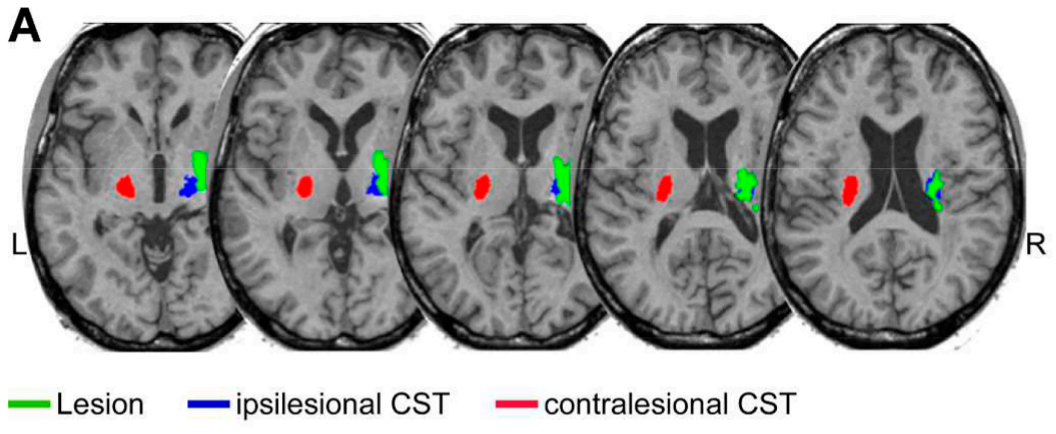

B

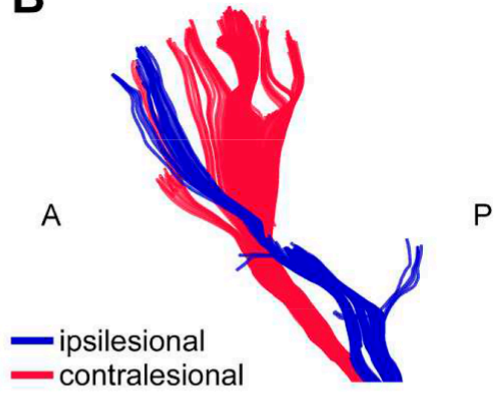

C

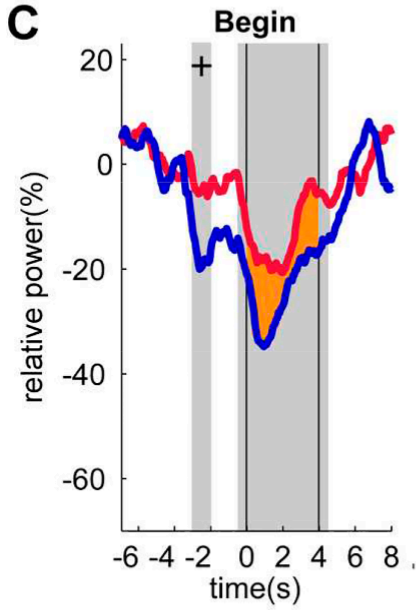

D

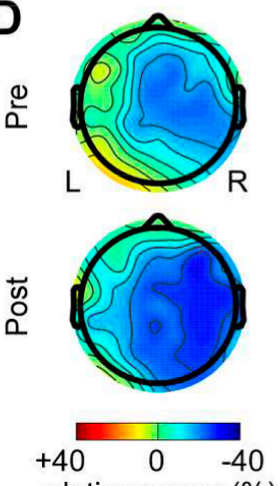

relative power (\%)
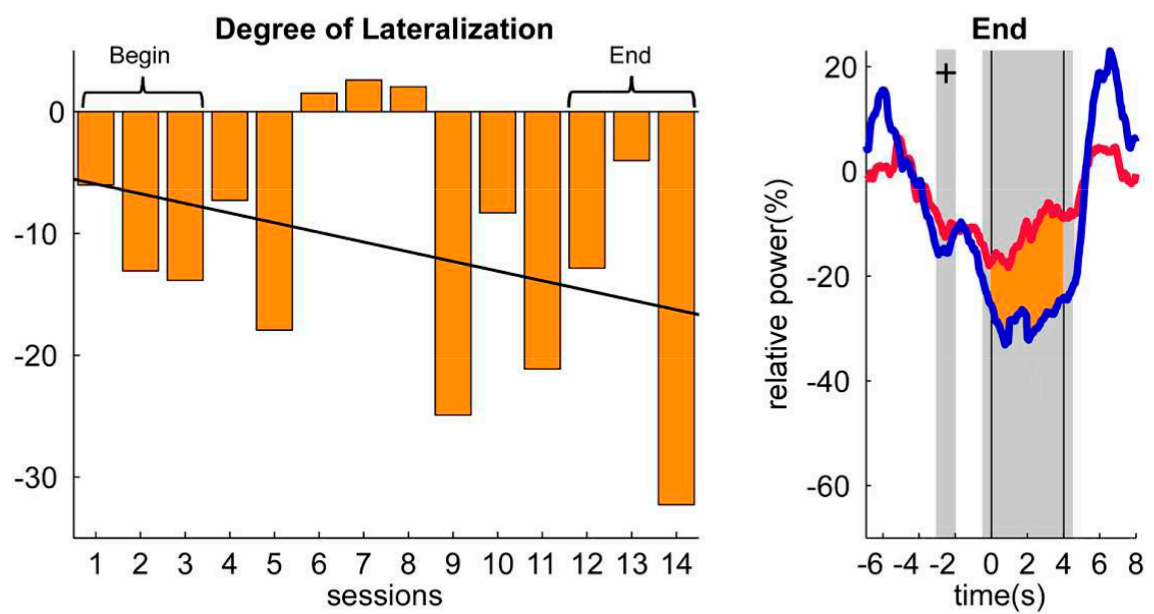

E

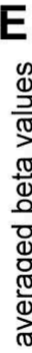

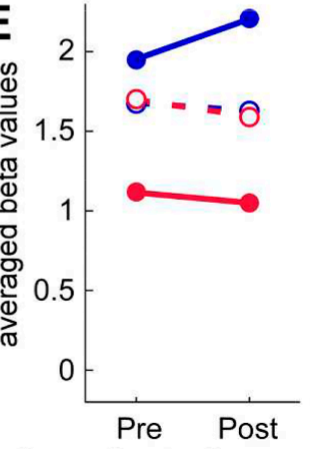

Sensorimotor Area ipsilesional

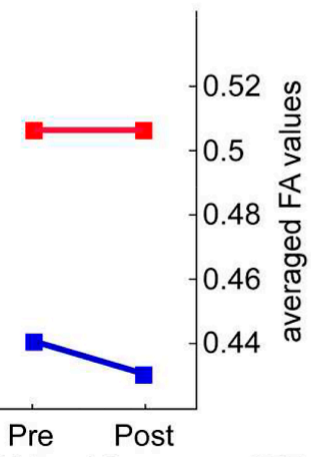

OVisual Area $\quad$ CST - contralesional

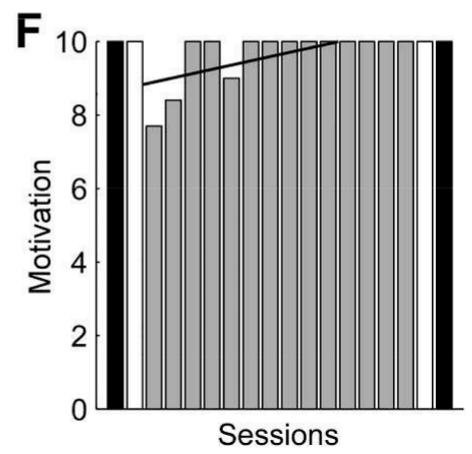

- fMRI $\quad$ IHD EEG पLD EEG

Figure 3. Lesion characteristics and secondary outcome measures $P 21$. As Figure 2, but $(A)$ shows the axial slices corresponding to $z=47$, $52,57,62$, and 67 in MNI (Montreal Neurological Institute) space.

although fMRI sessions revealed increased sensorimotor BOLD activity for both hemispheres (Figure 4E). In line with fMRI changes, increased FA could be observed for both CSTs (Figure 4E). P22 was highly motivated throughout the study, although his motivation seemed to be higher in the first half (bootstrapping 1000; $P>.1$ ). On average, he rated the MI as intense and the NF as supportive, spontaneously he said, "The feedback blocks are less effortful than the blocks without feedback." For both NF dimensions, good single trial classification accuracies were obtained across all training sessions (contralateral ERD, $M=78.19 \%, S D=8.57 \%$; ERD laterality, $M=75.47 \%, S D=3.98 \%$ ).

Taken together, no changes in motor function, as assessed by the mFMA and MAS, could be observed. MI of the affected hand was related to strong ERD, whereby the ERD lateralization increased over the course of training. AM induced only a 

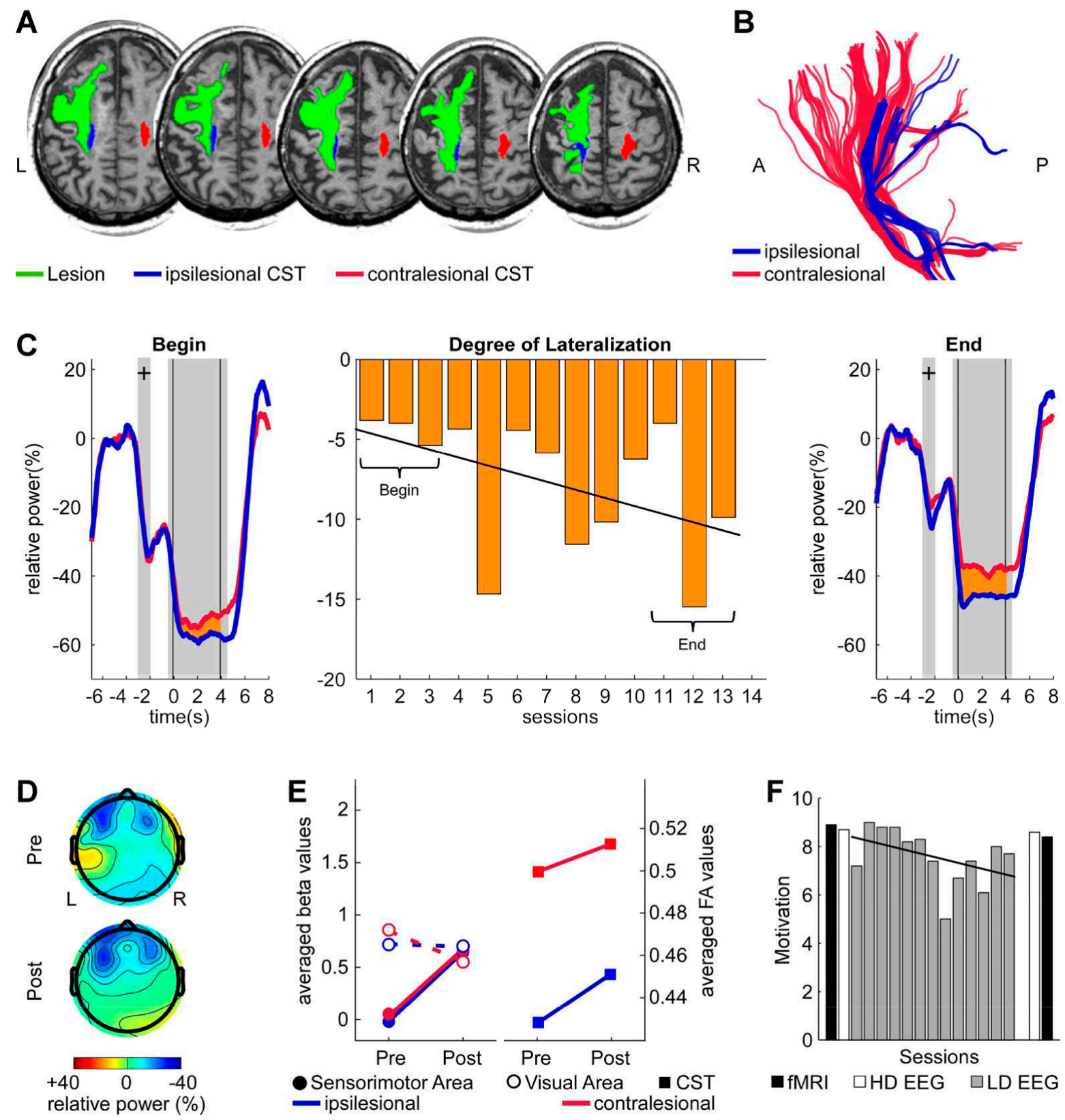

Figure 4. Lesion characteristics and secondary outcome measures $P 22$. As Figure 2, but $(A)$ shows the axial slices corresponding to $z=90$, 94, 98, 102, and 106 in MNI (Montreal Neurological Institute) space.

very subtle ERD and BOLD activity, however while ERD remained unchanged, fMRI activity was found to be increased after the MI training. On the structural level, an increase in white matter integrity was found.

\section{Discussion}

We examined the feasibility of conducting long-term MI EEGbased NF training at 3 stroke patients' homes and explored possible improvements on upper limb motor functioning. The protocol was employed in 3 chronic stroke patients with different lesion types and residual movement capacity. Training effects were evaluated by detailed motor assessment. Moreover, several secondary outcome measures were applied, including MI-induced ERD, AM-induced ERD, fMRI BOLD activity, and structural changes of CST white matter integrity. Over the course of MI EEG-based NF training, a remarkable increase in EEG lateralization during MI of the affected hand was observed 
in 1 patient (P20), with a smaller, yet nonsignificant increase in the other 2 patients (P21, P22). In 1 patient (P20) the EEG lateralization increase was accompanied by an increased lateralization of fMRI activity, a rebalanced CST integrity and improved motor abilities.

A key primary objective of this study was to investigate the feasibility of this training regime and identify potential problems of regular home-based MI NF training for stroke patients with chronic motor impairments. Specifically, we expected potential disturbances in executing the training due to daily life demands, the cognitive demands of this NF implementation and potential interferences of the home-based setting on EEG recording quality. Overall, we noted a very positive response to the training. Comments that were made spontaneously by patients indicated that they liked the training and the challenge associated with it. Moreover, they did not report adverse effects of the EEG application and did not struggle with the demanding training regime. All 3 individuals were very motivated, cooperative, and able to perform MI and to process the NF. Although this observation seems to be consisted across several patient characteristics, for example, age and severity of motor disabilities, future investigations need to evaluate the applicability of this NF protocol for stroke patients with cognitive deficits. Moreover, as evaluated by visual inspection of the EEG raw data, good EEG data quality was obtained at the patients' homes. Taken together, this provides solid evidence that frequent home-based MI NF training in patients with chronic motor disabilities is feasible.

Over the course of training, changes in cortical activation related to MI of the affected hand were observed on a descriptive level in all 3 patients. Specifically, an increase in lateralization was evident. Increased activity in sensorimotor regions of the contralesional hemisphere related to movement attempts of the paretic limb has been frequently documented after stroke. ${ }^{11,23,24,27-31}$ Moreover, the resulting reduction in cortical lateralization has been related to poor recovery. ${ }^{23}$ Along the same lines, shifts in laterality toward an increased ratio of ipsilesional to contralesional sensorimotor cortex activity have been related to changes in behavioral performance. ${ }^{11,32}$ In our study, the patient with the strongest change in lateralization over the course of the training also showed significant motor improvement. In addition, ERD and fMRI BOLD activity induced by AM were more lateralized after than before the training. Finally, white matter integrity of the ipsilesional and contralesional CST were less distinct after the training. These functional and structural changes accompanied clinically relevant improvement of motor functions. In the absence of a control group we cannot argue for a causal relationship between MI NF training and the observed functional, structural, and behavioral changes, but our results clearly suggest that the employed training contributed to adaptive reorganization and motor recovery in this case. To which extent the observed recovery-related changes are due to the MI NF training, physical therapy or other factors remains speculative. For the 2 more severely affected patients, the training resulted only in changes on the descriptive level and no behavioral improvement could be observed. It is conceivable that the necessary amount of training to achieve clinically relevant changes is related to the degree of motor impairment. It could also be argued, that due to location and size of the lesion, or the overlap between lesion and CST, those patients are less likely to benefit from this type of training. However, all patients had preserved CST fibers and were able to imagine the instructed movement and both aspects seem necessary for MI training to be effective. Moreover, it is conceivable that a flexible training regime that is to some degree adapted to each patient leads to stronger improvements. Individually tailored training may include individualized hardware and software, for example, EEG caps ${ }^{33}$ and NF parameters, ${ }^{34}$ as well as an individualized training plan, which comprises not only the frequency of training but also the time of day at which it is conducted, the type and modality of NF and the imagined movement.

The main objective of the pre-post measurements was to investigate in depth the effects of home-based MI NF training. Particular emphasis was placed on evaluating the effects of MI NF training on the neural signatures of AM using highdensity EEG and fMRI. Broadly speaking, the cortical lateralization of movement-related activity assessed during the training sessions, pre-post fMRI and pre-post high-density EEG did not only yield converging results. Some discrepancies might originate from differences between MI and AM, differences in hemodynamic and electrophysiological recordings and normal variation that occurs between sessions. Regarding the first of these 3 aspects, MI was practiced during the training sessions and transfer effects on AM were evaluated in the pre-post comparison. Yet MI and AM activation patterns differ to some degree,$^{35}$ hence care should be taken when comparing the neuronal correlates of the training sessions and the pre-post measurements. The rehabilitative potential of MI training depends, among other factors, on a better understanding of the transfer from mentally practiced movements to overt physical performance. On the second aspect, we recently revealed a clear and consistent dissociation between hemodynamic and electrophysiological signatures regarding motor execution- and MI-induced cortical lateralization using concurrent EEG-fMRI and EEGfunctional near-infrared spectroscopy recordings. ${ }^{17,36,37}$ These indicate that lateralization patterns obtained in one imaging modality cannot be easily transferred to a second independent imaging modality, which may contribute to the dissociation between fMRI BOLD activity and ERD measures obtained in the present investigation. Among others, this illustrates how important multimodal neuroimaging studies are to develop a comprehensive picture of functional recovery. In addition, the intra-individual variance that was evident in the training sessions highlights the importance of permanent monitoring. Rehabilitation progress should be ideally assessed based on performance trends and not only on single sessions, such as pre-post comparisons, as the latter can be strongly influenced by intra-individual variance in performance. For these reasons, direct comparisons between sessions and imaging modalities should be made cautiously. 


\section{Conclusion}

This work demonstrates for the first time that frequent homebased MI EEG-based NF training is feasible in chronic stroke patients. First anecdotal evidence also indicates that our training regime has the potential to facilitate behavioral, functional, and structural changes. Future research will be required to optimize the training regime and verify its effectiveness in a proper clinical trial study.

\section{Acknowledgments}

We are grateful to I. Arizanovic for implementing the changes in the neurofeedback.

\section{Author Contributions}

All authors contributed to the concept and deisgn of the study. CZ and CS collected the data; CZ, AS and CK analysed and interpreted the data. All authors wrote and reviewd the manuscript.

\section{Declaration of Conflicting Interests}

The author(s) declared no conflicts of interest with respect to the research, authorship, and/or publication of this article.

\section{Funding}

The author(s) disclosed receipt of the following financial support for the research, authorship, and/or publication of this article: This work was supported by the $\mathrm{PhD}$ program "Signals and Cognition" (Niedersächsisches Ministerium für Wissenschaft und Kultur) (CZ), by grant KR 3433/2-1 of the German Research Foundation (DFG) (CK, JM), and Task Group 7 of the Cluster of Excellence Hearing4all (SD).

\section{Supplementary Material}

Supplementary material for this article is availabe online.

\section{References}

1. Jones TA, Adkins DL. Motor system reorganization after stroke: stimulating and training toward perfection. Physiology (Bethesda). 2015;30:358-370.

2. Murray CJL, Vos T, Lozano R, et al. Disability-adjusted life years (DALYs) for 291 diseases and injuries in 21 regions, 1990-2010: a systematic analysis for the Global Burden of Disease Study 2010. Lancet. 2012;380:2197-2223.

3. Hankey GJ, Jamrozik K, Broadhurst RJ, Forbes S, Anderson CS. Long-term disability after first-ever stroke and related prognostic factors in the Perth Community Stroke Study, 1989-1990. 2002;33:1034-1040.

4. Jeannerod M. Neural simulation of action: a unifying mechanism for motor cognition. Neuroimage. 2001;14:103-109.

5. Cicinelli P, Marconi B, Zaccagnini M, Pasqualetti P, Filippi MM, Rossini PM. Imagery-induced cortical excitability changes in stroke: a transcranial magnetic stimulation study. Cereb Cortex. 2006; 16:247-253.

6. Langhorne P, Coupar F, Pollock A. Motor recovery after stroke: a systematic review. Lancet Neurol. 2009;8:741-754.

7. Page SJ, Levine P, Leonard AC. Effects of mental practice on affected limb use and function in chronic stroke. Arch Phys Med Rehabil. 2005;86:399-402.
8. Crosbie JH, McDonough SM, Gilmore DH, Wiggam MI. The adjunctive role of mental practice in the rehabilitation of the upper limb after hemiplegic stroke: a pilot study. Clin Rehabil. 2004;18:60-68.

9. Liu KP, Chan CC, Lee TM, Hui-Chan CW. Mental imagery for promoting relearning for people after stroke: a randomized controlled trial. Arch Phys Med Rehabil. 2004;85:1403-1408.

10. Grosse-Wentrup M, Mattia D, Oweiss K. Using brain-computer interfaces to induce neural plasticity and restore function. $J$ Neural Eng. 2011;8:025004.

11. Buch E, Weber C, Cohen LG, et al. Think to move: a neuromagnetic brain-computer interface (BCI) system for chronic stroke. Stroke. 2008;39:910-917.

12. Broetz D, Braun C, Weber C, Soekadar SR, Caria A, Birbaumer N. Combination of brain-computer interface training and goaldirected physical therapy in chronic stroke: a case report. Neurorehabil Neural Repair. 2010;24:674-679.

13. Caria A, Weber C, Brötz D, et al. Chronic stroke recovery after combined BCI training and physiotherapy: a case report. Psychophysiology. 2011;48:578-582.

14. Ramos-Murguialday A, Broetz D, Rea M, et al. Brain-machine interface in chronic stroke rehabilitation: a controlled study. Ann Neurol. 2013;74:100-108.

15. Shindo K, Kawashima K, Ushiba J, et al. Effects of neurofeedback training with an electroencephalogram-based brain-computer interface for hand paralysis in patients with chronic stroke: a preliminary case series study. J Rehabil Med. 2011;43:951-957.

16. Pichiorri F, Morone G, Petti M, et al. Brain-computer interface boosts motor imagery practice during stroke recovery. Ann Neurol. 2015;77:851-865.

17. Zich C, Debener S, Kranczioch C, Bleichner MG, Gutberlet I, De Vos M. Real-time EEG feedback during simultaneous EEG-fMRI identifies the cortical signature of motor imagery. Neuroimage. 2015;114:438-447.

18. Debener S, Minow F, Emkes R, Gandras K, De Vos M. How about taking a low-cost, small, and wireless EEG for a walk? Psychophysiology. 2012;49:1617-1621.

19. Kranczioch C, Zich C, Schierholz I, Sterr A. Mobile EEG and its potential to promote the theory and application of imagery-based motor rehabilitation. Int J Psychophysiol. 2014;91:10-15.

20. De Vos M, Kroesen M, Emkes R, Debener S. P300 speller BCI with a mobile EEG system: comparison to a traditional amplifier. J Neural Eng. 2014;11:036008.

21. Debener S, Emkes R, De Vos M, Bleichner M. Unobtrusive ambulatory EEG using a smartphone and flexible printed electrodes around the ear. Sci Rep. 2015;5:16743.

22. Renard Y, Lotte F, Gibert G, et al. OpenViBE : an open-source software platform to design, test, and use brain-computer interfaces in real and virtual. Presence. 2010;19:35-53.

23. Ward NS, Brown MM, Thompson AJ, Frackowiak RSJ. Neural correlates of outcome after stroke: a cross-sectional fMRI study. Brain. 2003;126:1430-1448.

24. Ward NS, Brown MM, Thompson AJ, Frackowiak RSJ. Neural correlates of motor recovery after stroke: a longitudinal fMRI study. Brain. 2003;126:2476-2496.

25. Fugl-Meyer AR, Jääskö L, Leyman I, Olsson S, Steglind S. The post-stroke hemiplegic patient. 1. A method for evaluation of physical performance. Scand J Rehabil Med. 1975;7:13-31.

26. Pandyan AD, Johnson GR, Price CI, Curless RH, Barnes MP, Rodgers H. A review of the properties and limitations of the 
Ashworth and modified Ashworth Scales as measures of spasticity. Clin Rehabil. 1999;13:373-383.

27. Johansen-Berg H, Rushworth MFS, Bogdanovic MD, Kischka U, Wimalaratna S, Matthews PM. The role of ipsilateral premotor cortex in hand movement after stroke. Proc Natl Acad Sci U S A. 2002;99:14518-14523.

28. Feydy A, Carlier R, Roby-Brami A, et al. Longitudinal study of motor recovery after stroke: recruitment and focusing of brain activation. Stroke. 2002;33:1610-1617.

29. Cramer SC, Nelles G, Benson RR, et al. A functional MRI study of subjects recovered from hemiparetic stroke. Stroke. 1997;28:2518-2527.

30. Chollet F, DiPiero V, Wise RJ, Brooks DJ, Dolan RJ, Frackowiak RS. The functional anatomy of motor recovery after stroke in humans: a study with positron emission tomography. Ann Neurol. 1991;29:63-71

31. Caramia MD, Iani C, Bernardi G. Cerebral plasticity after stroke as revealed by ipsilateral responses to magnetic stimulation. Neuroreport. 1996;7:1756-1760.

32. Calautti C, Leroy F, Guincestre JY, Marié RM, Baron JC. Sequential activation brain mapping after subcortical stroke: changes in hemispheric balance and recovery. Neuroreport. 2001;12:3883-3886.
33. Zich C, De Vos M, Kranczioch C, Debener S. Wireless EEG with individualized channel layout enables efficient motor imagery training. Clin Neurophysiol. 2015;126:698-710.

34. Blankertz B, Losch F, Krauledat M, Dornhege G, Curio G, Müller $\mathrm{K}-\mathrm{R}$. The Berlin brain-computer interface: accurate performance from first-session in BCI-naïve subjects. IEEE Trans Biomed Eng. 2008;55:2452-2462.

35. Blokland Y, Spyrou L, Thijssen D, et al. Combined EEG-fNIRS decoding of motor attempt and imagery for brain switch control: an offline study in patients with tetraplegia. IEEE Trans Neural Syst Rehabil Eng. 2014;22:222-229.

36. Zich C, Debener S, Kranczioch C, Chen L-C, De Vos M. Lateralization patterns for movement execution and imagination investigated with concurrent EEG-fMRI and EEG-fNRIS. In: Müller-Putz GR, Huggins JE, Steyrl D, eds. Proceedings of the Sixth International Brain-Computer Interface Meeting: BCI Past, Present, and Future, Pacific Grove, California, USA. Graz, Austria: Verlag der Technischen Universität Graz; 2016:101.

37. Zich C, Debener S, Thoene A-K, Chen L-C, Kranczioch C. Simultaneous EEG-fNIRS reveals how age and feedback affect motor imagery signatures. Neurobiol Aging. 2017;49:183-197. 